
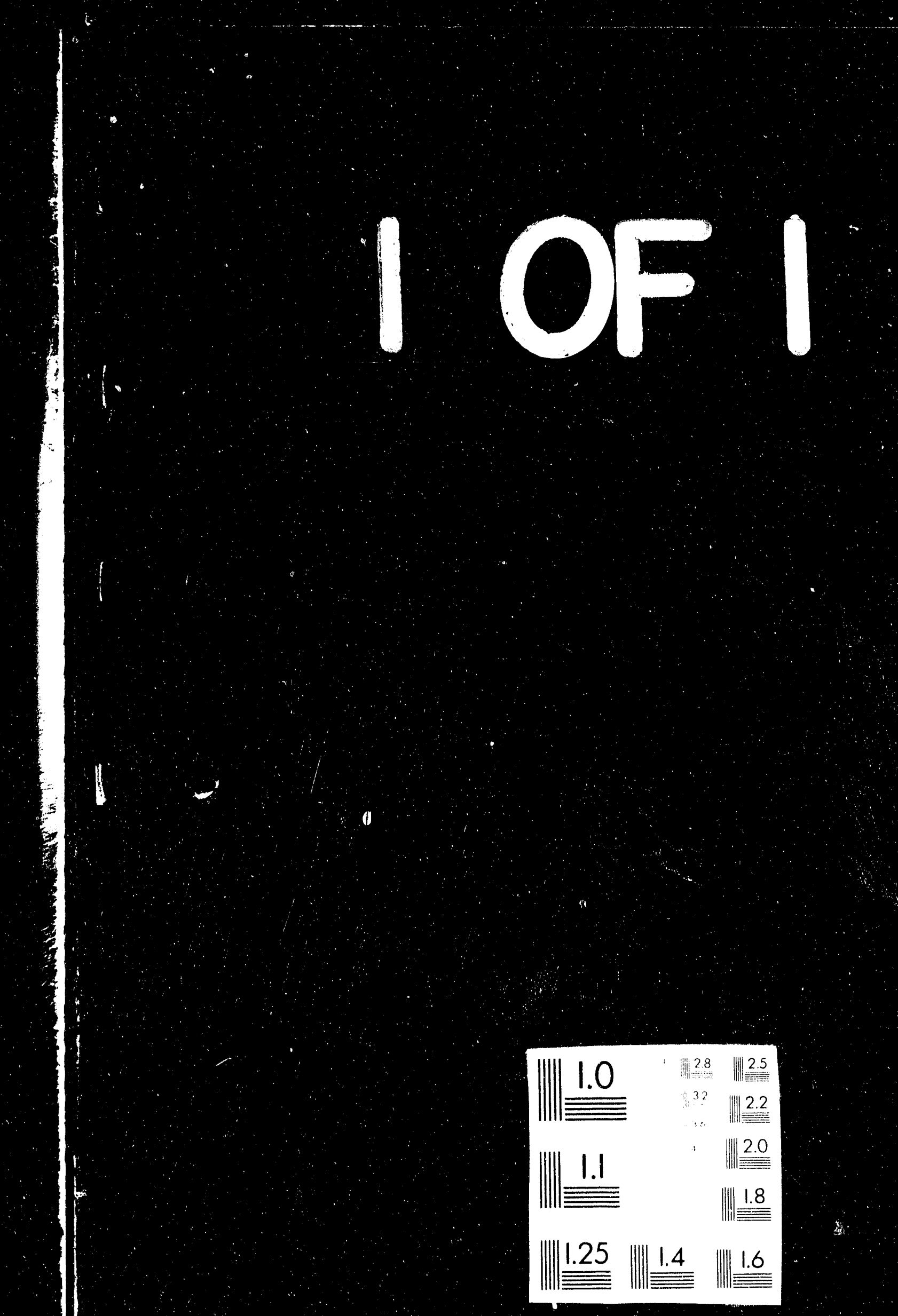


\title{
ANALYZING GIGAHERTZ BUNCH LENGTH INSTABILITIES WITH A DIGITAL SIGNAL PROCESSOR*
}

\author{
Robert E. Stege, Jr., Patrick Krejcik, Michiko G. Minty \\ Stanford Linear Accelerator Center, Stanford University, Stanford, C.A 94309
}

\begin{abstract}
A bunch length instability, nicknamed the "sawtooth", because of its transient behavior, has been observed at high current running in the Stanford Linear Collider (SLC) electron damping ring. The incompatibility of this instability with successful SLC running prompted its study using a high bandwidth real-time spectrum analyzer, the Tektronix 3052 digital signal processor (DSP) system. This device has been used to study energy ramping in storage rings ${ }^{1}$ but this is the first time it has been used to study transient instability phenomena. It is a particularly valuable tool for use in understanding non-linear, multiple frequency phenomena. The frequency range of this device has been extended through the use of radio frequency (RF) down converters. This paper describes the measurement setup and presents some of the results.
\end{abstract}

\section{INTRODUCTION}

In the SLC north (electron) and south (positron) damping rings, both the longitudinal and the transverse phase space of the orbiting particles is radiation damped to achieve smaller beam emittances for injection into the linear accelerator (LINAC). During normal running, the beams are injected and extracted at $120 \mathrm{~Hz}$ allowing a total time for damping of about $8.3 \mathrm{msec}$ for the electrons and $16.6 \mathrm{msec}$ for the positrons. In 1991, as SLC beam currents were increased to produce more luminosity and increase $\mathrm{Z}$ production, "fliers" began to interfere with operation. A "flier" was an errant beam pulse, with a transverse displacement, which resulted in beam trips due to excessive radiation levels. In 1992, as currents were increased further, an instability was observed that involved a blow-up of the bunch length and energy spread in the damping rings. This instability, nicknamed the "sawtooth", was visible on an oscilloscope when the bunch length signal, obtained from a BPM sum signal, repeatedly blew up and then became radiation damped. The sawtooth instability had a measured single bunch threshold of about $3 \times 10^{10}$ particles. For running above this threshold the

* Work supported by the Department of Energy, contract DE-AC03-76SF00515.

Contributed to the 1992 Accelerator Instrumentation Workshop,
Berkeley, CA, October 27-30,1992 
instability was overcome by ramping the RF gap voltage from the nominal $800 \mathrm{kV}$ to about $250 \mathrm{kV}$. In anticipation of running higher currents in the 1993 running period, it is expected that the instability may prove difficult to suppress and therefore should be understood.

Measurements made with a network analyzer showed that the beam was easily excited at three times the synchrotron tune, $v_{s}$. The synchrotron frequency and hence the excitation at $3 v_{s}$ was also observed to depend on beam current. The optimum frequency for observing an $\mathrm{m}^{\text {th }}$ order synchrotron sideband is approximately

$$
f=\frac{m+1}{2 \pi \sigma_{L}} \times c,
$$

where: $\quad \sigma_{L}=$ bunch length $\left(\cong 8.5 \mathrm{~mm}\right.$ at $\left.3 \times 10^{10}\right)$

$c=$ speed of light $=3.0 \times 10^{10} \mathrm{~cm} / \mathrm{sec}$

as determined by where the $\mathrm{m}^{\text {th }}$ order Bessel function which describes the envelope of the beam frequency spectrum peaks. Thus we expect to be maximally sensitive to the $3 v_{s}$ sideband near $25 \mathrm{GHz}$.

\section{FUNCTIONAL DESCRIPTION OF THE TEKTRONIX 3052}

A conventional analog spectrum analyzer mixes the input signal with a swept local oscillator. The swept IF is then applied to a fixed filter and the output of the filter used to drive a CRT display of the frequency spectrum. This technique has an inherent disadvantage when analyzing multiple frequency transient phenomena. Due to this sweeping technique, different frequencies are measured at different times. The signals that we are interested in have low signal to noise ratios requiring slow sweep speeds to resolve thus making it difficult to observe transient events. A DSP spectrum analyzer, on the other hand, is a real time analyzer which uses a parallel digital filter bank to simultaneously analyze the range of frequencies and produce a digital frequency spectrum. Since the DSP processes all frequencies simultaneously, transient phenomena may be studied and correlations between signals observed.

A block diagram of the Tektronix 3052 DSP system is shown in figure 1. The input signal enters through an analog front end module which contains the necessary gain and attenuation controls as well as the analog to digital converter (ADC). The ADC provides 10 bit resolution at each data point and operates at $25.6 \mathrm{MHz}$. This digitized signal is then passed to the parallel filter bank.

The parallel filter bank has 1024 filters or "bins" which are equally spaced in the frequency domain. The filter outputs are sampled sequentially 


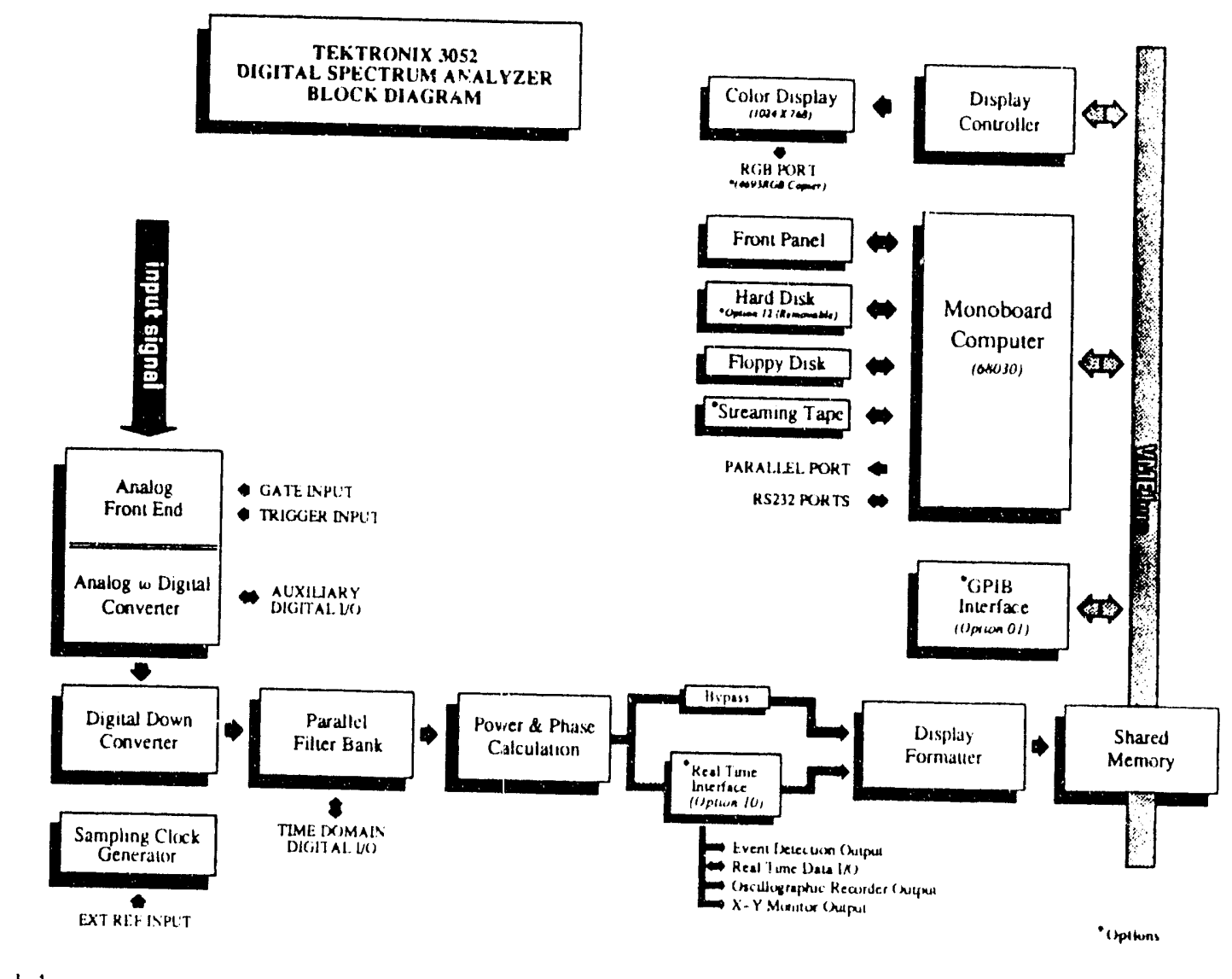

Te'ktroniX 3052 OPERATORS MANUAL.

Figure 1. Block Diagram of the Tektronix 3052 DSP System. ${ }^{2}$

at a maximum rate of $5 \mathrm{kHz}$ and the central 801 are used to represent one spectral frame. The frequency resolution of the 3052 is determined by the frequency span divided by the 801 filter bins. Our frequency range of interest typically spanned 1 or $2 \mathrm{MHz}$ corresponding to a bin resolution of 1.25 and $2.50 \mathrm{kHz}$ respectively. For these ranges, the criteria for real time analysis, that the sample time be at least twice the resolution, is satisfied. The default filters are of a linear-phase, finite impulse response design. In addition the user may choose from Bartlett, Blackman, Hamming, Hanning or rectangular filters. User designed filters can also be implemented in the system.

The real and imaginary data is then converted back to amplitude and phase form and formatted for the display. The data is displayed on a 16 inch color monitor with 1024 pixel by 768 line resolution. The available displays include amplitude vs. frequency, phase vs. frequency, a color spectrogram and a waterfall display. The color spectrogram format, which displays frequency vs. time with color coded amplitude, is particularly useful for observing transient phenomena. In the data to be presented the bottom half shows a color spectrogram with the vertical axis indicating time with the most recent event at the bottom. Frequency is plotted on the horizontal axis and the amplitudes are color coded according to the scale as indicated on the 
right. The upper plot shows the frequency vs. amplitude corresponding to the spectral frame indicated by the small arrowhead in the left margin of the color spectrogram below. This arrow can be moved through the entire spectrogram making it possible to review the data frame by frame.

Because frame update rates as high as $5 \mathrm{MHz}$ are not always necessary or desirable, the 3052 is provided with several display summary modes which can be adjusted by varying the Display Reduction Rate or $\mathrm{R}$ value. Options include a display of every $R^{\text {th }}$ frame, the average of $R$ frames, the maximum of $R$ frames or the minimum and maximum over $R$ frames.

\section{MEASUREMENT SETUP}

The measurement setup is shown in figure 2. The signal from the beam was picked up on a single beam position monitor (BPM) stripline electrode located in the electron damping ring. To minimize cable losses, a high frequency, 1/4 inch heliax cable was run directly through the roof of the vault for a total cable length of less than $20 \mathrm{~m}$. The initial processing was done using a HP 70000 series modular spectrum analyzer with a $100 \mathrm{~Hz}-26.5 \mathrm{GHz}$ HP 70909A RF Section. In the data to be presented the center frequency of the $\mathrm{HP}$ spectrum analyzer was set to a harmonic of the revolution frequency. A center frequency near $20 \mathrm{GHz}$ was chosen to be close to the calculated frequency of maximum sensitivity and less than the roll off frequency of the

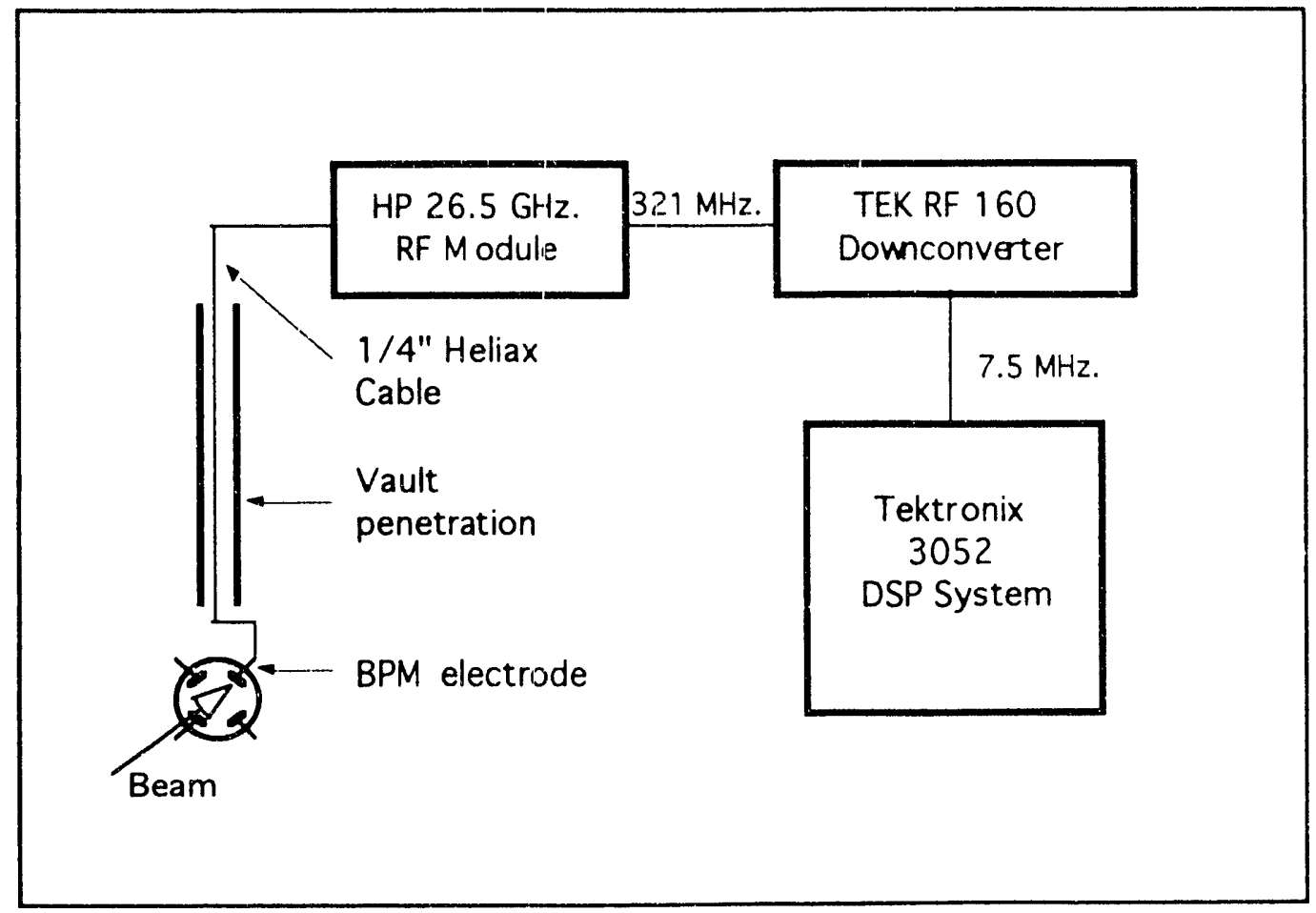

Figure 2. Measurement setup. 
heliax cable. The analyzer was set to zero span and the sweep was stopped. In this mode the analyzer was functionally equivalent to a receiver. The spectrum analyzer converted the input signal to a $321.4 \mathrm{MHz}$ IF signal which was then mixed down to $7.5 \mathrm{MHz}$ using a Tektronix RF162 down converter. This $7.5 \mathrm{MHz}$ signal was then transported to the Tektronix 3052 DSP system for processing.

The Tektronix 3052 was set for proper input gain and reference level offset with the aid of the "thermometer" in the lower right hand corner of the display. The thermometer indicated the input level from 0 to 100 percent of the full range of the ADC. The DSP was remotely triggered on successive beam pulses in the ring. The trigger timing could be varied to allow the beam spectrum to be recorded at different times in the machine store cycle. The display reduction rate was adjusted to obtain the desired time scale on the color spectrogram.

\section{RESULTS}

Figure 3 shows the measured beam spectrum with the DSP triggered $1 / 2 \mathrm{msec}$ after injection. The center frequency in the figure is $20.349 \mathrm{GHz}$ down converted to $7.5 \mathrm{MHz}$ and the span is $5 \mathrm{MHz}$.

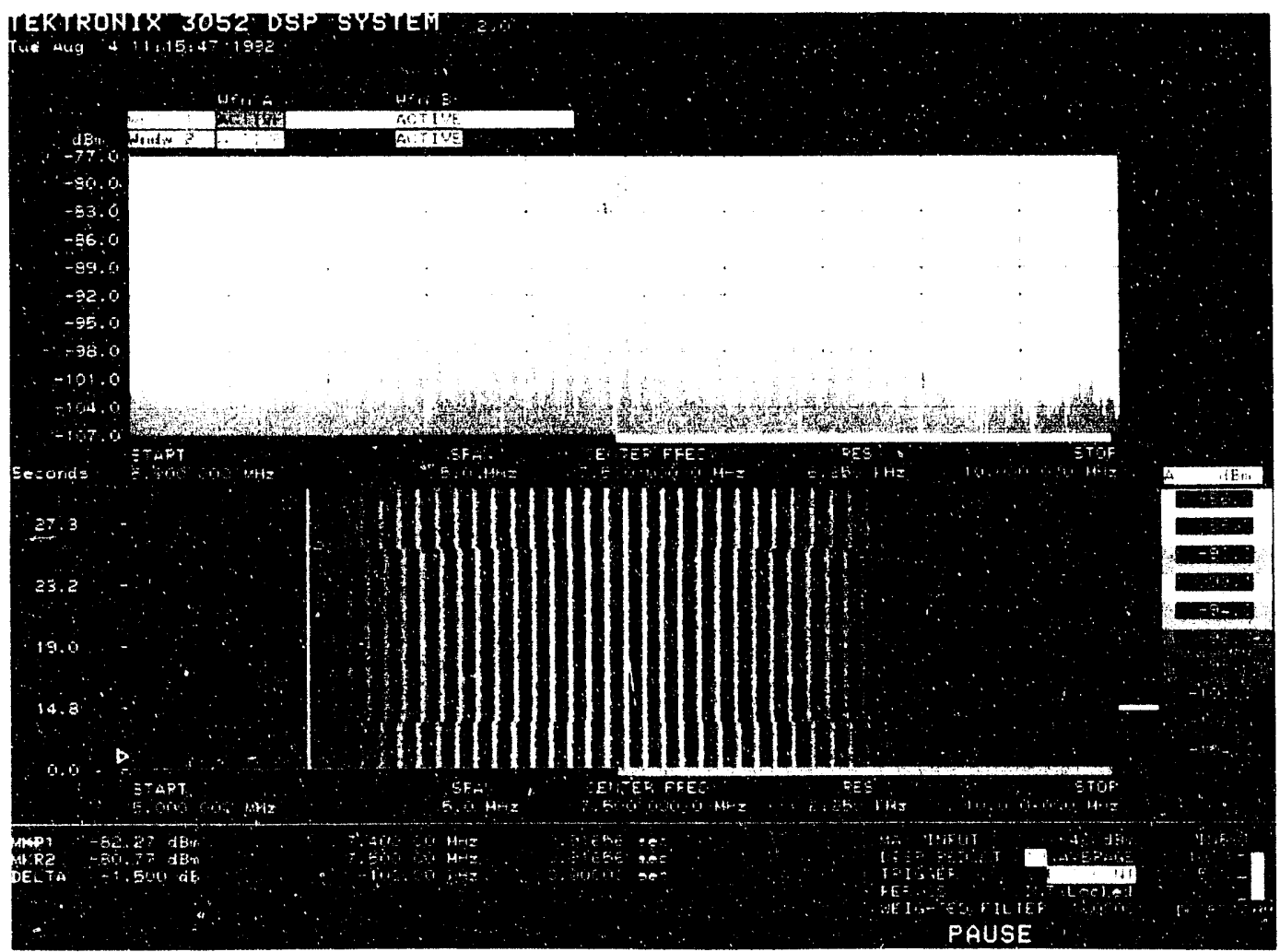

Figure 3. Many higher order sidebands are visible when the beam is recorded at injection time. 
From the spectrum one can observe many sidebands separated by about $100 \mathrm{kHz}$, the synchrotron frequency. The observation of at least 15 sidebands shows that this set up was very sensitive to the frequency range of interest. The rf voltage was varied slightly to confirm that the side bands were derived from the beam and not an instrument artifact. For the signal to be beam related the sidebands are separated by $v_{S} \propto V_{R F}^{42}$

Shown in figure 4 is the measured beam spectrum as a function of current where the beam was stored in the ring for over 200 seconds. The center frequency was $20.349 \mathrm{GHz}$, the span was $1.0 \mathrm{MHz}$ and the DSP was triggered externally and synchronously with the injection triggers. The display reduction was set to display every $120^{\text {th }}$ trigger.

The frequency vs. amplitude plot shows again the behavior at injection. The color spectrogram shows the behavior after injection. With currents above the instability threshold, the sidebands, characteristic of the instability, were pronounced. As the current decayed, the power in the sidebands decreased. When the current decayed to below the instability threshold, the $3 v_{s}$ sidebands were observed to disappear.

Shown in figure 5 is the measured spectrum as a function of $\mathrm{rf}$ cavity gap voltage. The beam current was $3.6 \times 10^{10}$ particles per bunch and the

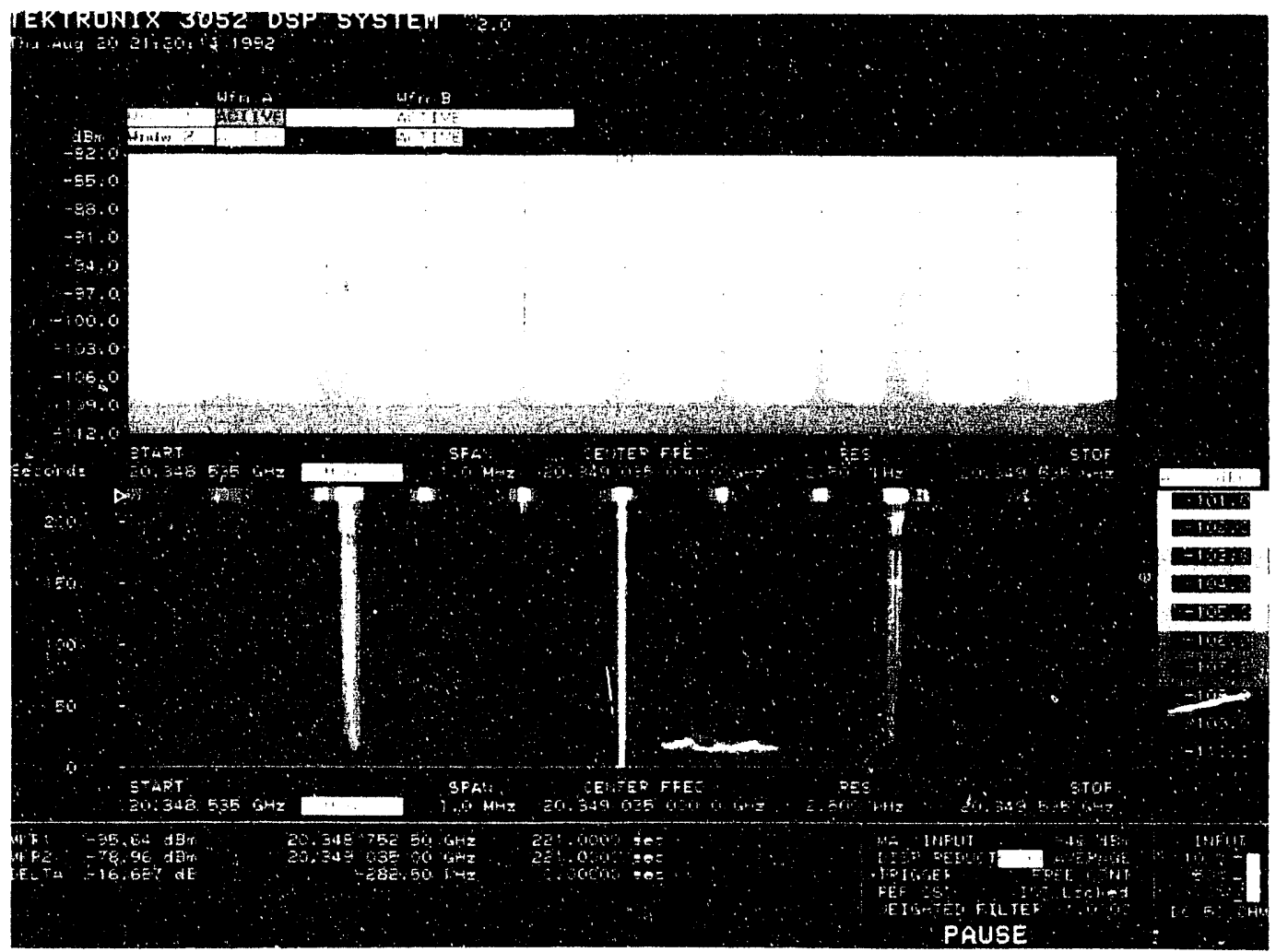

Figure 4. Showing the disappearance of the $3 v_{s}$ sideband as the current drops below the instability threshold. 
machine cycle time was $8.3 \mathrm{~ms}$. The rf cavity voltage was varied from $990 \mathrm{kV}$ to $550 \mathrm{kV}$. The renter frequency was $20.349 \mathrm{GHz}$ down converted to $7.5 \mathrm{MHz}$ and the span was $2 \mathrm{MHz}$. The DSP was triggered externally with the trigger delay adjusted to view the beam close to extraction. The display reduction was set to 25. The time scale of the color spectrogram spans about 40 minutes. The data acquisition was paused each time the rf voltage was adjusted.

Shown in the frequency vs. amplitude spectrum is the beam response with the cavity voltage set to $990 \mathrm{kV}$. At these high currents synchrotron sidebands were clearly discerned at both $3 v_{s}$ and $6 v_{s}$. This was a direct indication of higher order longitudinal microbunching modes. In the color spectrogram the beam response was measured with the cavity voltage set as indicated on the left. As the voltage was lowered the power in each side band decreased. At $550 \mathrm{kV}$, the instability was no longer excited.

\section{CONCLUSION}

The DSP has proved invaluable in discriminating the high frequency mode structure associated with instabilities in high intensity, bunched beams. In combination with a high frequency receiver we observed bunch structure

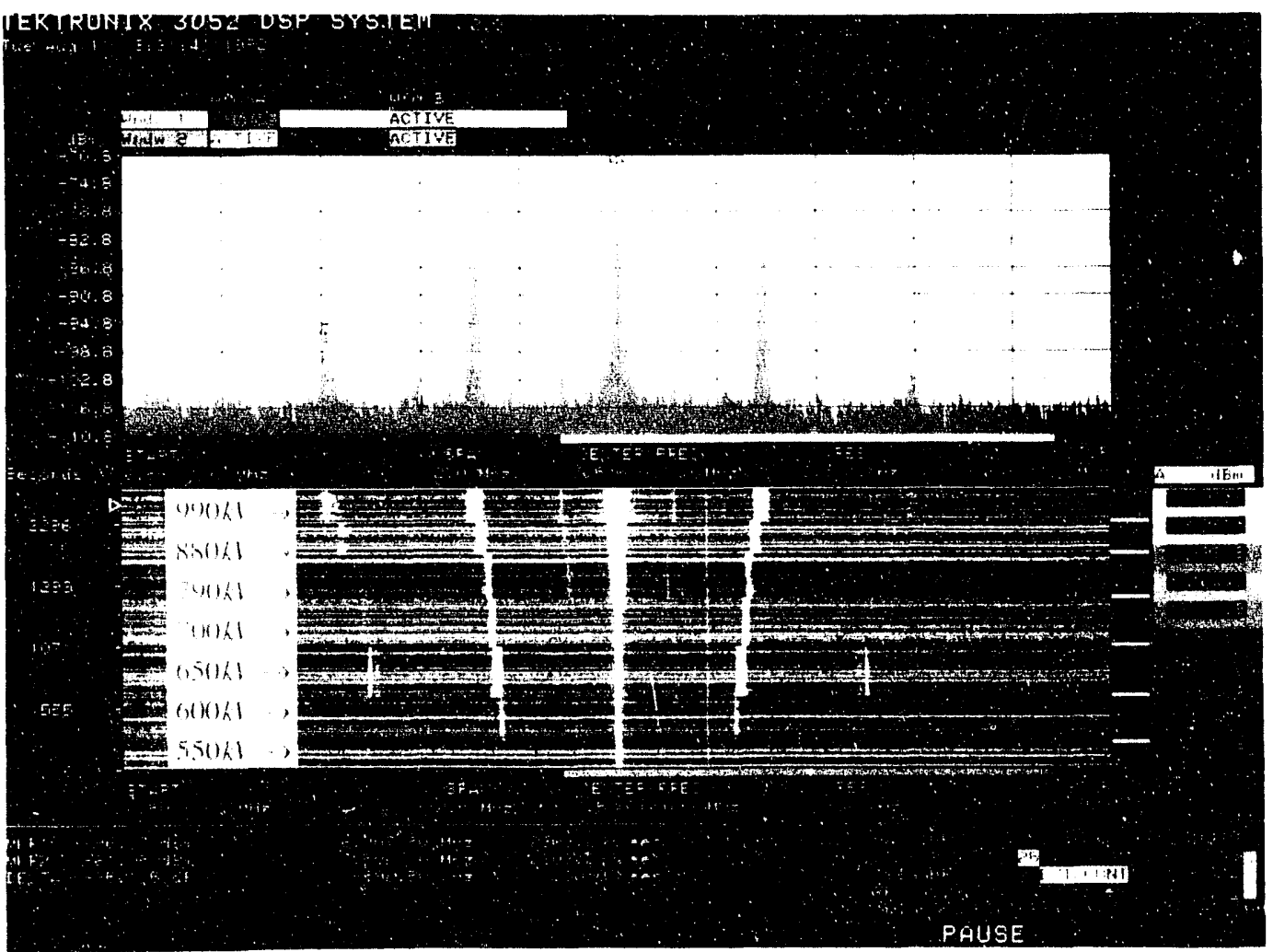

Figure 5. The $3 v_{s}$ and $6 v_{s}$ sideband frequencies for different RF voltages. 
in the $20 \mathrm{GHz}$ regime. The fine time resolution of the DSP allowed the high frequency modes to be identified with specific dynamic phenomena during
different parts of the beam store cycle.

\title{
ACKNOWLEDGMENTS
}

The authors gratefully acknowledge Marc Ross and Doug McCormick of SLAC for help in setting up and performing these experiments. In addition we thank Kevin Cassidy and Ken Andrew of Tektronix for their technical advice. Thanks are also due to Flemming Pedersen of CERN and Robert Siemann of SLAC who encouraged us to look in this frequency regime for signatures of bunch length instabilities.

\section{REFERENCES}

1 Kevin J. Cassidy, Sam Howry, "Development Of A Model For Ramping In A Storage Ring," AIP Conference Proceedings No 252, page 144 (1991).

2 Reprinted with permission from Tektronix DSP Operators Manual, Part No. 070-6494-02, Product Group 2M, page 1-4 (1990).

\section{DISCLAIMER}

\begin{abstract}
This report was prepared as an account of work sponsored by an agency of the United States Government. Neither the United States Government nor any agency thereof, nor any of their employees, makes any warranty, express or implied, or assumes any legal liability or responsibility for the accuracy, completeness, or usefulness of any information, apparatus, priduct, or process disclosed, or represents that its use would not infringe privately owned rights. Reterence herein to any specific commercial product, process, or service by trade name, trademark, manufacturer, or otherwise does not necessarily constitute or imply its endorsement, recommendation, or favoring by the United States Government or any agency thereof. The views and opinions of authors expressed herein do not necessarily state or reflect those of the United States Government or any agency thereof.
\end{abstract}



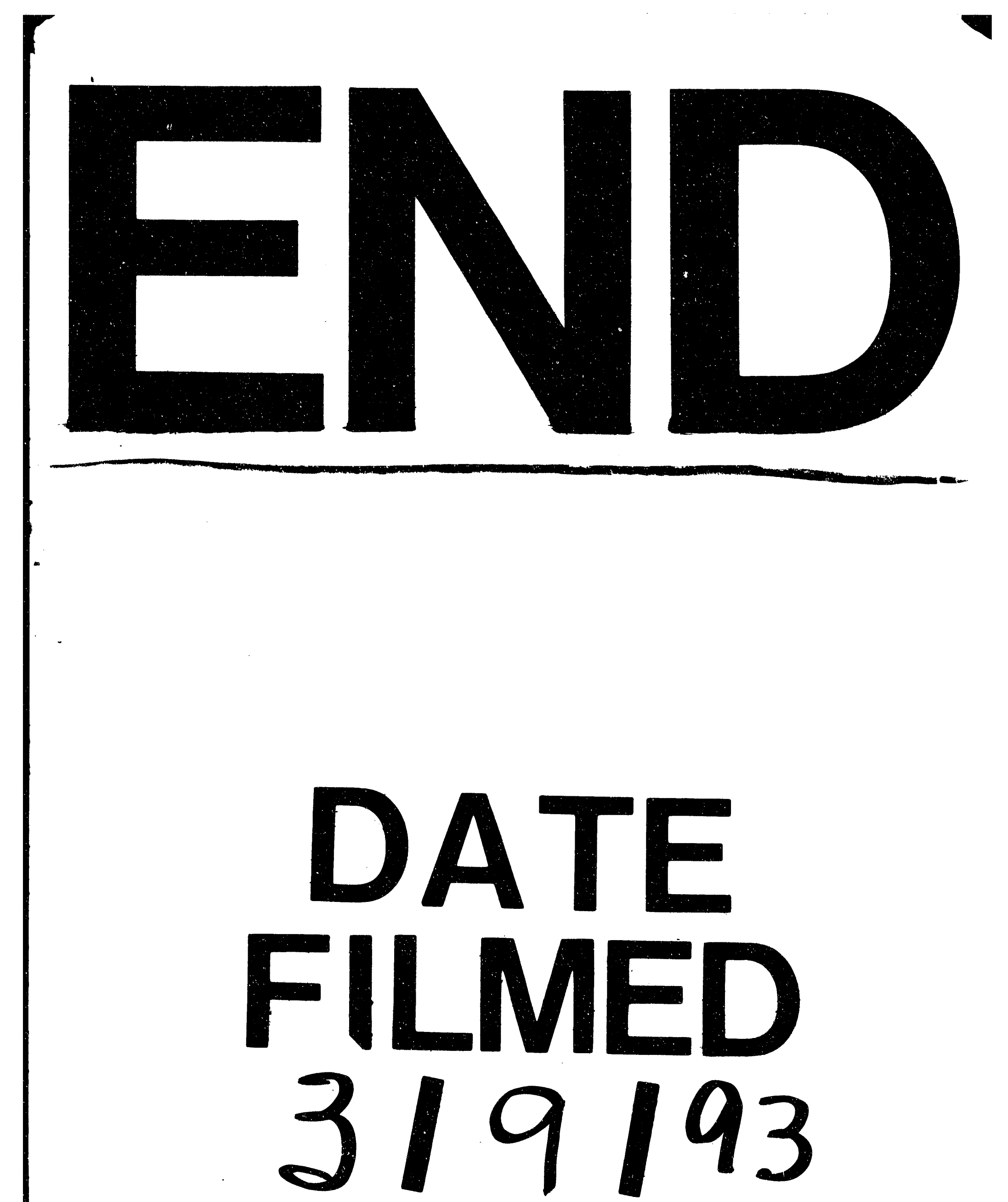
